\title{
Influenza Virus Usurps an Interferon-Induced Translational Program to Promote Viral Replication ${ }^{\dagger}$
}

\author{
Mitchell P. Ledwith 1, Vy Tran 1, Thiprampai Thamamongood 2,3,4, Christina A. Higgins 1, \\ Shashank Tripathi 5,6, Max W. Chang 7, Christopher Benner 7, Adolfo García-Sastre 5,6,8,9, \\ Martin Schwemmle 2,10, Adrianus C. M. Boon ${ }^{11}$, Michael S. Diamond ${ }^{11}$ and Andrew Mehle ${ }^{1, *}$ \\ 1 Medical Microbiology and Immunology, University of Wisconsin Madison, Madison, WI 53706-1521, USA; \\ ledwith@wisc.edu (M.P.L.); vgtran@wisc.edu (V.T.); cahiggins3@wisc.edu (C.A.H.) \\ 2 Faculty of Medicine, University of Freiburg, 79106 Freiburg, Germany; \\ thiprampai.thamamongood@sgbm.uni-freiburg.de (T.T.); martin.schwemmle@uniklinik-freiburg.de (M.S.) \\ 3 Spemann Graduate School of Biology and Medicine, University of Freiburg, 79104 Freiburg, Germany \\ 4 Faculty of Biology, University of Freiburg, 79104 Freiburg, Germany \\ 5 Department of Microbiology, Icahn School of Medicine at Mount Sinai, New York, NY 10029-5674, USA; \\ shashank.tripathi@mssm.edu (S.T.); adolfo.garcia-sastre@mssm.edu (A.G.-S.) \\ 6 Global Health and Emerging Pathogens Institute, Icahn School of Medicine at Mount Sinai, New York, \\ NY 10029-5674, USA \\ 7 Department of Medicine, University of California, San Diego, San Diego, CA 92093, USA; \\ mchang@ucsd.edu (M.W.C.); cbenner@ucsd.edu (C.B.) \\ 8 Department of Medicine, Division of Infectious Diseases, Icahn School of Medicine at Mount Sinai, \\ New York, NY 10029-5674, USA \\ 9 The Tisch Cancer Institute, Icahn School of Medicine at Mount Sinai, New York, NY 10029-6574, USA \\ ${ }^{10}$ Institute of Virology, Medical Center, University of Freiburg, D-79104 Freiburg, Germany \\ 11 Departments of Medicine, Molecular Microbiology, and Pathology \& Immunology, \\ Washington University School of Medicine, St. Louis, MO 63110, USA; jboon@wustl.edu (A.C.M.B.); \\ diamond@wusm.wustl.edu (M.S.D.) \\ * Correspondence: amehle@wisc.edu \\ † Presented at Viruses 2020-Novel Concepts in Virology, Barcelona, Spain, 5-7 February 2020.
}

Published: 21 July 2020

\begin{abstract}
Hosts mount prudently tuned responses to viral infection in an attempt to block nearly every step of the replication cycle. Viruses must adapt to replicate in this hostile antiviral cellular state. Interferon stimulation or pathogen challenge robustly induces expression of IFIT (interferoninduced proteins with tetratricopeptide repeats) proteins. IFITs are a family of proteins that bind RNA and play antiviral roles during infection. Thus, we were surprised to identify the IFIT family as top candidate proviral host factors for influenza A virus (IAV) in a genome-wide CRISPR-Cas9 knockout screen. We validated the proviral activity of IFIT2 by showing that IFIT2-deficient cells support lower levels of IAV replication and exhibit defects in viral gene expression. The molecular functions of IFIT2, let alone how they are used by influenza virus, are unknown. Using CLIP-seq, we showed that IFIT2 binds directly to viral and cellular mRNAs in AU-rich regions largely in the 3'UTR, with a preference for a subset of interferon-stimulated mRNAs. IFIT2 also associates with actively translating ribosomes in infected cells to facilitate the translation of viral messages. IFIT2responsive elements from an IAV mRNA were sufficient to confer translational enhancement to exogenous transcripts in cis. Conversely, mutation of these elements or the use of an IFIT2 RNAbinding mutant ablated stimulation of viral gene expression. Together, these data link the RNAbinding capability of IFIT2 to changes in translational efficiency of target viral mRNAs and the stimulation of viral replication. They establish a model for the normal function of IFIT2 as an antiviral protein affecting the post-transcriptional fate of cellular mRNAs and explain how influenza virus repurposes IFIT2 to support viral replication. Our work highlights a new node for the
\end{abstract}


regulation of translation during interferon responses and highlights how canonical antiviral responses may be repurposed to support viral replication.

Keywords: influenza; translation; RNA; post-transcriptional regulation

(C) 2020 by the authors. Licensee MDPI, Basel, Switzerland. This article is an open access article distributed under the terms and conditions of the Creative Commons Attribution (CC BY) license (http://creativecommons.org/licenses/by/4.0/). 